Energy Research Journal 1 (2): 165-170, 2010

ISSN 1949-0151

(C) 2010 Science Publications

\title{
Stress-Strain Analysis of Abdominal Aortic Wall: A Case of 3D Geometry Simulation
}

\author{
P. Khamdaengyodtai, P. Sakulchangsatjatai and P. Terdtoon \\ Department of Mechanical Engineering, Faculty of Engineering, \\ Chiang Mai University, Chiang Mai, Thailand
}

\begin{abstract}
Problem statement: This study analyzed stress-strain of abdominal aortic wall in 3D geometry simulation. Abdominal aorta is a portion of the aorta vessel in the abdomen which many cardiovascular diseases often occur. Approach: Abdominal aortic wall was considered as 1 layers-3 dimensional cylindrical geometry and homogeneous, incompressible and anisotropic material. Deformation of aortic wall was elastic deformation. Numerical method was used in our simulation. The abdominal aorta was under luminal loading of pressure in physiological pressure range of mice. Experimental parameters from previous study were used as initial configuration of present study. To verify present simulation, the simulation results were compared with the experimental data from previous study. Results: From the simulation, the wall thickness decreased, whereas the diameter, lumen cross-sectional area increased with internal pressures. The magnitude of radial stress, longitudinal stress and circumferential stress increased with increasing internal pressure and values were largest at the inside surface. The radial stress was obviously compressive and varied from a value equal to the negative of the internal pressure at the inside to zero at the outside. Conclusion: Stressstrain analysis of abdominal aortic wall in a case of 3D geometry simulation can be simulated using numerical model. This model had been verified with experimental data from previous study. Good agreement was obtained. Mathematical model which considering aortic wall by using finite element method could be beneficially used as addition data for medical diagnosis.
\end{abstract}

Key words: Three dimension simulation, abdominal aorta, stress, strain

\section{INTRODUCTION}

In blood circulation system, the aorta acts as both conduit and an elastic chamber. The elastic of aorta serves to convert the heart's pulsatile flow to steady flow in peripheral vessels. The structural component of vessel wall are altered various conditions such as aging, diabetes and hypertension. That will be a risk factor for arteriosclerosis due to the mechanical properties abnormality through various mechanisms. One of the implications of the structural changes is the change in mechanical properties of the blood vessel. Increased stiffness of large arteries represents an early risk factor for cardiovascular diseases. Therefore, the assessment of aortic mechanical properties is particularly important in understanding the mechanisms of cardiovascular diseases. The major goal of present study is to analyze stress-strain of abdominal aortic wall in a case of threedimensional geometry simulation. Such that stiffness can be accordingly determined.

\section{MATERIALS AND METHODS}

Mathematical model of abdominal aortic wall: Abdominal aorta geometry in present study was considered three-dimensional thick-walled cylindrical vessel. The abdominal aorta was under statics internal pressure which is from flowing blood pressure acted on inside wall of abdominal aorta. In macroscopic study of aorta, abdominal aorta can be assumed as homogeneous, isotropic and incompressible material. Internal pressure acted on inside wall cause of inflation of abdominal aorta. Experimental parameters from study of Guo and Kassab (2003) Humphrey and Delange (2004); Silver et al. (2003) and Zhao et al. (2002) were used as initial configuration of present study. As abdominal aorta had no internal pressure, outside diameter of $0.2945 \mathrm{~mm}$, wall thickness of 44.467 micron, elastic modulus of $70 \mathrm{kPa}$, poisons ratio of 0.49 were considered. Internal pressures as in mice physiological pressure range of 30,60, 90, 120 and $150 \mathrm{mmHg}$ were studied.

Corresponding Author: P. Khamdaengyodtai, Department of Mechanical Engineering, Faculty of Engineering, Chiang Mai University, Thailand 50200 Tel: +66-53-944144/ext. 911 Fax: +66-53-226014 
Theoretical equations: Considering inflation of threedimensional geometry abdominal aorta as in Fig. 1, plan strain had been suitably used. Plane strain is defined to be a state of strain in which the strain normal to the radial-longitudinal ( $\mathrm{r}-\mathrm{z})$ plane and the shear stains $\gamma_{r \theta}$ and $\gamma_{\theta z}$ were assumed to be zero. Assumptions of plane strain are realistic for long vessel (in the $\mathrm{z}$ direction) with constant cross-sectional area subjected to internal pressure that act only in the radial direction and do not vary in the longitudinal direction. In axisymmatric, the radial displacements developed circumferential strains that induced stresses $\sigma_{\mathrm{r}}, \sigma_{\theta}, \sigma_{\mathrm{z}}$ and $\gamma_{\mathrm{rz}}$, where $\mathrm{r}, \theta$ and $\mathrm{z}$ indicated the radial, circumferential and longitudinal directions, respectively. Components of stress related to a cylindrical coordinate system were shown in Fig. 2, using the standard notation $\sigma_{\text {(face)(direction). Triangular }}$ torus elements were used to idealize the axisymatric system because they can be used to simulate complex surfaces.

Because of symmetry about the longitudinal axis, the stresses were independent of the $\theta$ coordinate (no torsion was considered). Therefore, all derivatives with respected to $\theta$ vanished and the displacement component, $\mathrm{v}$ (tangent to the $\theta$ direction), the shear strains, $\gamma_{\mathrm{r} \theta}$ and $\gamma_{\theta \mathrm{z}}$ and the shear stresses, $\tau_{\mathrm{r} \theta}$ and $\tau_{\theta \mathrm{z}}$ were all zero.

Strain-displacement relations in cylindrical coordinate system (Boresi and Chong, 1961; Bergel, 1961; Carroll, 1998; Darly, 2007) were:

$\begin{array}{cc}\varepsilon_{\mathrm{r}}=\frac{\partial \mathrm{u}}{\partial \mathrm{r}} & \varepsilon_{\theta}=\frac{\mathrm{u}}{\mathrm{r}} \\ \varepsilon_{\mathrm{z}}=\frac{\partial \mathrm{w}}{\partial \mathrm{z}} & \gamma_{\mathrm{rz}}=\frac{\partial \mathrm{u}}{\partial \mathrm{z}}+\frac{\partial \mathrm{w}}{\partial \mathrm{r}}\end{array}$

The isotropic stress-strain relationship, obtained by simplifying the general stress-strain relationships was:

$$
\left\{\begin{array}{c}
\sigma_{\mathrm{r}} \\
\sigma_{\mathrm{z}} \\
\sigma_{\theta} \\
\tau_{\mathrm{rz}}
\end{array}\right\}=\frac{\mathrm{E}}{(1+v)(1-2 v)}\left[\begin{array}{cccc}
1-v & v & v & 0 \\
v & 1-v & v & 0 \\
v & v & 1-v & 0 \\
0 & 0 & 0 & \frac{1-2 v}{2}
\end{array}\right]\left\{\begin{array}{c}
\varepsilon_{\mathrm{r}} \\
\varepsilon_{\mathrm{z}} \\
\varepsilon_{\theta} \\
\gamma_{\mathrm{rz}}
\end{array}\right\}
$$

Numerical method: The finite element method is numerical procedure which can model the behavior of structure of vessel. Analytical solutions generally require the solution of ordinary or partial differential equations, which, because of the complication geometry, loading and material properties, are not usually obtainable. Hence, we need to rely on numerical methods, such as finite element method, for acceptable solutions. Derivation of the strain triangular element stiffness matrix and equations followed these steps.

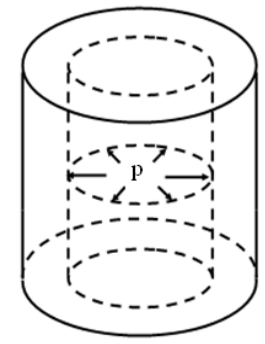

Fig. 1: Thick-walled cylindrical vessel subjected to internal pressure

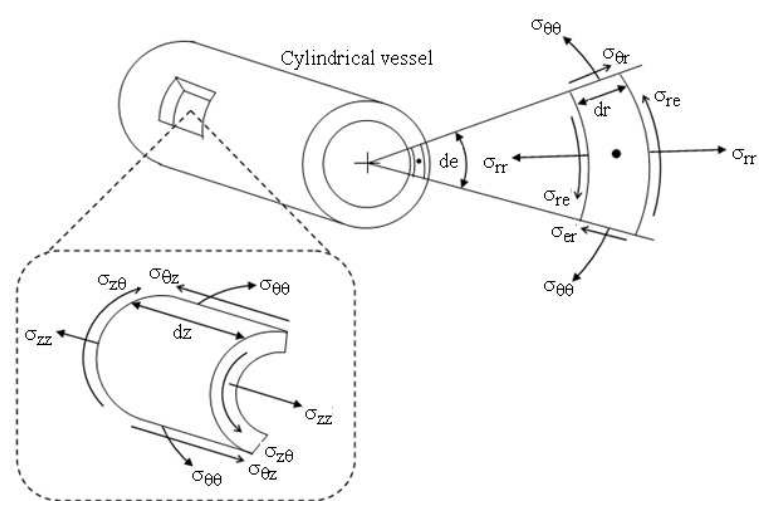

Fig. 2: Components of stress relative to a cylindrical coordinate system

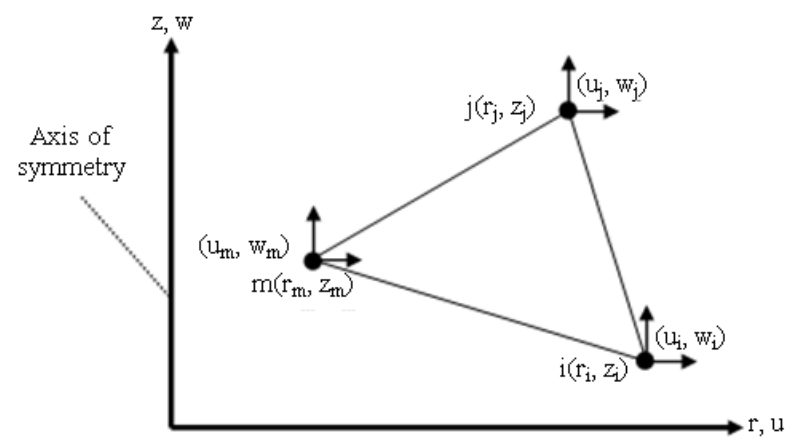

Fig. 3: Triangular element showing degree of freedom

Select element type: Triangular elements as in Fig. 3 were employed because boundaries of irregularly shaped bodies can be closely approximated in this way and because the expressions related to the triangular element were comparatively simple.

Each node had two degrees of freedom, radial and longitudinal displacement. $\mathrm{u}_{\mathrm{i}}$ and $\mathrm{w}_{\mathrm{i}}$ represent the node $\mathrm{i}$ displacement components in the radial and longitudinal direction, respectively. Triangular elements of abdominal aorta and discretized cylinder slice were shown in Fig. 4 and 5. 


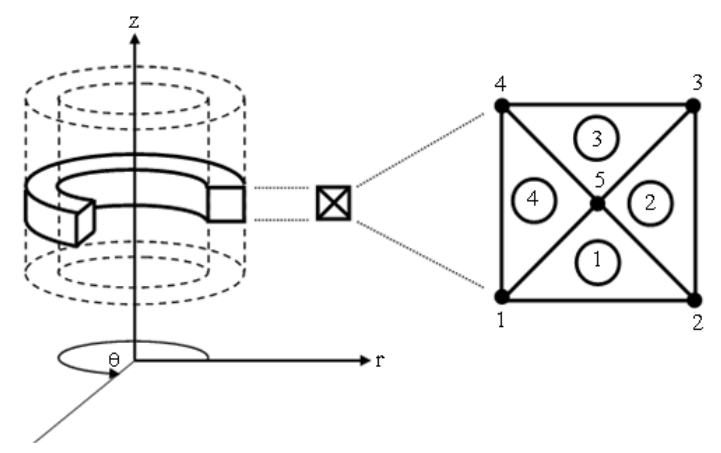

Fig. 4: Triangular elements of abdominal aorta

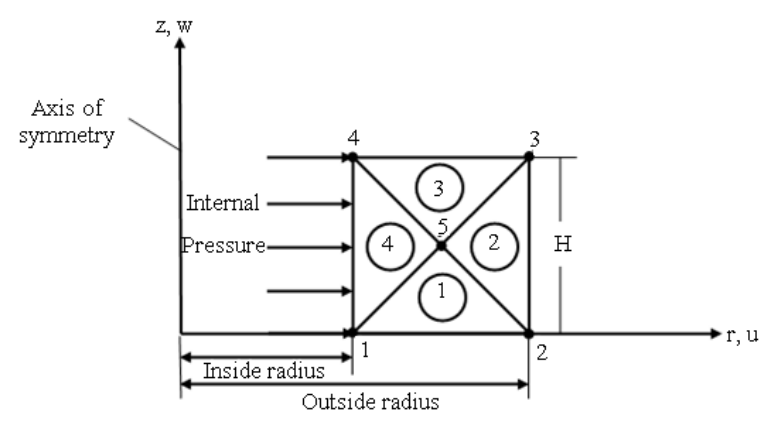

Fig. 5: Discretized cylinder slice

Select displacement functions: A linear displacement functions were taken to be:

$$
\begin{aligned}
& \mathrm{u}(\mathrm{r}, \mathrm{z})=\mathrm{a}_{1}+\mathrm{a}_{2} \mathrm{r}+\mathrm{a}_{3} \mathrm{z} \\
& \mathrm{w}(\mathrm{r}, \mathrm{z})=\mathrm{a}_{4}+\mathrm{a}_{5} \mathrm{r}+\mathrm{a}_{6} \mathrm{z}
\end{aligned}
$$

Equation 3 was then used to find finite element matrix. Expressing a set of Eq. 3 in abbreviated matrix form, that was:

$$
\{\psi\}=[\mathrm{N}]\{\mathrm{d}\} \mathrm{a}
$$

Where:

$\{\psi\}=$ The displacement function

$[\mathrm{N}]=$ The shape functions represented the shape of $\{\psi\}$

$\{\mathrm{d}\}=$ Displacement matrix

Nodal displacements can be calculated by Eq. 4 .

Define the strain-displacement and stress-strain relationships: Equation 1 were used and then rewritten in finite element matrix form as:

$$
\{\varepsilon\}=[B]\{d\}
$$

where, $[\mathrm{B}]$ is a function of the radial and longitudinal coordinates. Equation 2 was used and then rewritten in finite element matrix form as:

$\{\sigma\}=[\mathrm{D}][\mathrm{B}]\{\mathrm{d}\}$

where, [D] is given by the first matrix on the right side of Eq. 2. Strains of elements obtained from Eq. 5 were used to calculate stresses of elements.

Derive the element stiffness matrix and equations: Using principle of minimum potential energy, the equations can be generated for strain triangular element. The equation of system obtained by principle of minimum potential energy was:

$\iiint_{V}[B]^{\mathrm{T}}[\mathrm{D}][\mathrm{B}] \mathrm{dV}\{\mathrm{d}\}=\{\mathrm{f}\}$

It can be seen that the stiffness matrix was:

$[\mathrm{k}]=\iiint_{\mathrm{V}}[\mathrm{B}]^{\mathrm{T}}[\mathrm{D}][\mathrm{B}] \mathrm{dV}$

or

$[\mathrm{k}]=2 \pi \iint_{\mathrm{A}}[\mathrm{B}]^{\mathrm{T}}[\mathrm{D} \perp \mathrm{B}] \mathrm{rdrdz}$

Method which was used to evaluate Eq. 9 was from [B] evaluation by a centroidal point of element. by:

Surface force from internal pressure can be found

$\{\mathrm{f}\}=\iint_{\mathrm{S}}\left[\mathrm{N}_{\mathrm{s}}\right]^{\mathrm{T}}\{\mathrm{T}\} \mathrm{dS}$

Assemble the element equations: The element equations assembly was in order to obtain the global equations and introduce boundary conditions.

Solve for the nodal displacements: Nodal displacements were used in Eq. 5 to calculate strains of elements.

Solve for the elements forces (stresses): Strains of elements obtained by using Eq. 5 were used to calculate stresses of elements.

\section{RESULTS}

Results were shown in Fig. 6-11. To verify present simulation, the simulation results were compared with the experimental data from Guo and Kasseb (2003). 
Energy Rec. J. 1 (2): 165-170, 2010

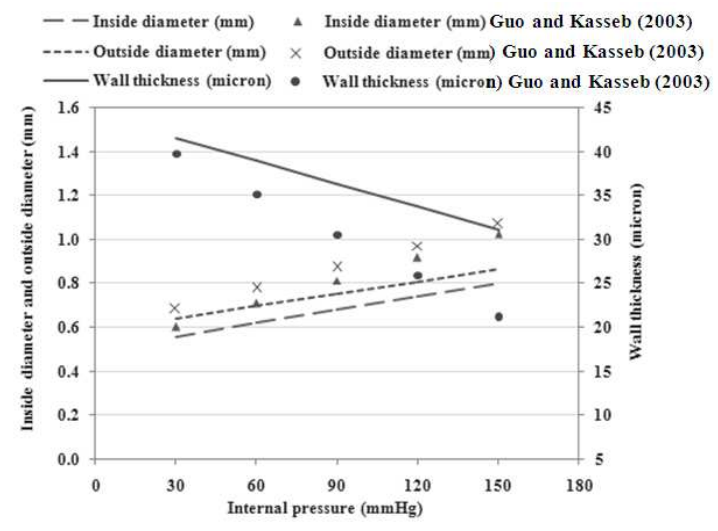

Fig. 6: Effect of internal pressure on inside diameter, outside diameter and wall thickness

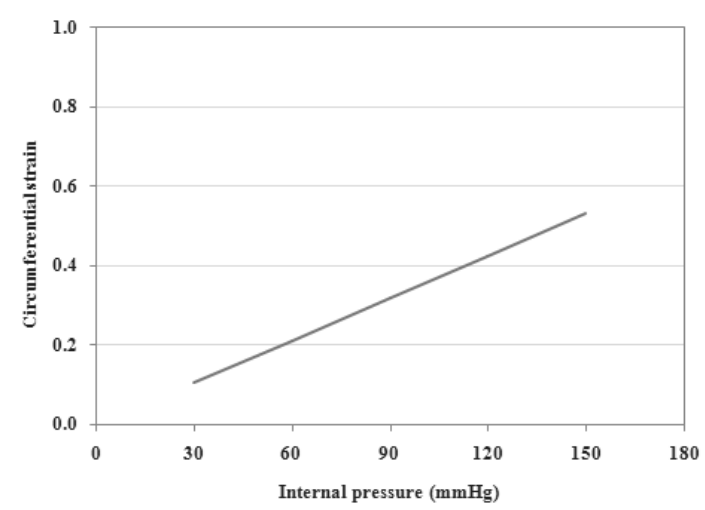

Fig. 7: Effect of internal pressure on circumferential strain

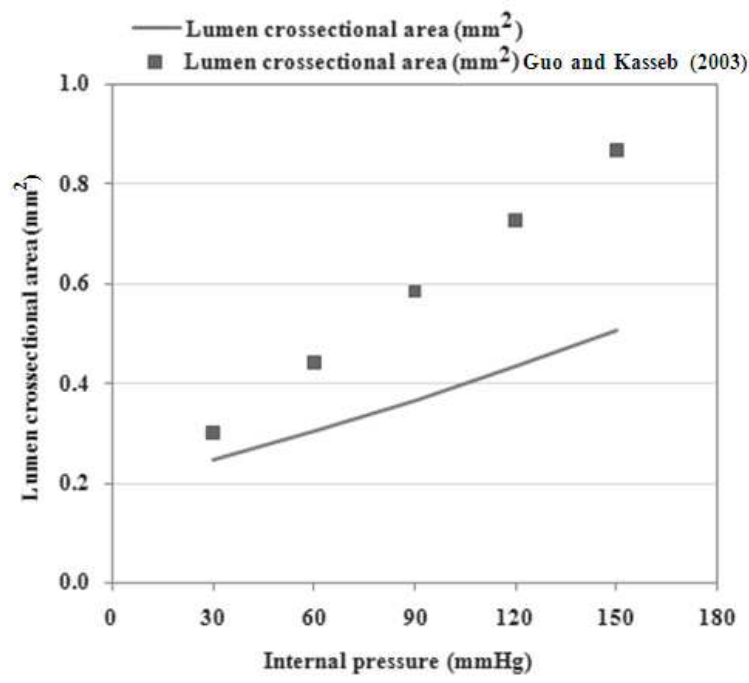

Fig. 8: Effect of internal pressure on lumen crosssectional area

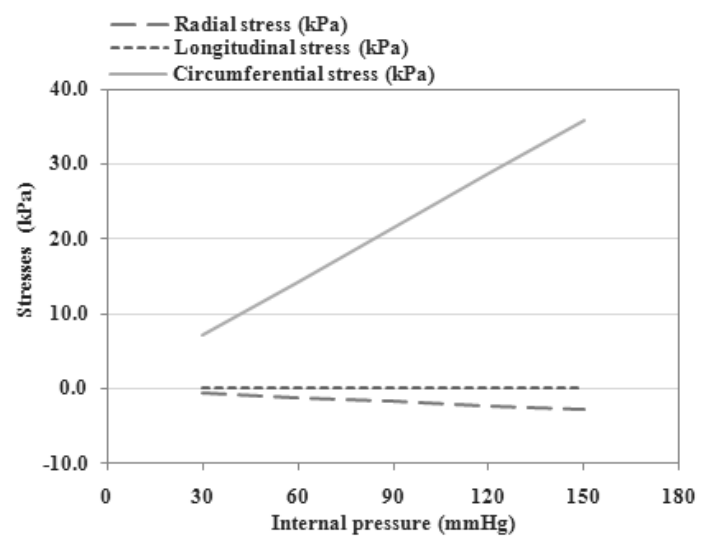

Fig. 9: Effect of internal pressure on radial stress, longitudinal stress and circumferential stress

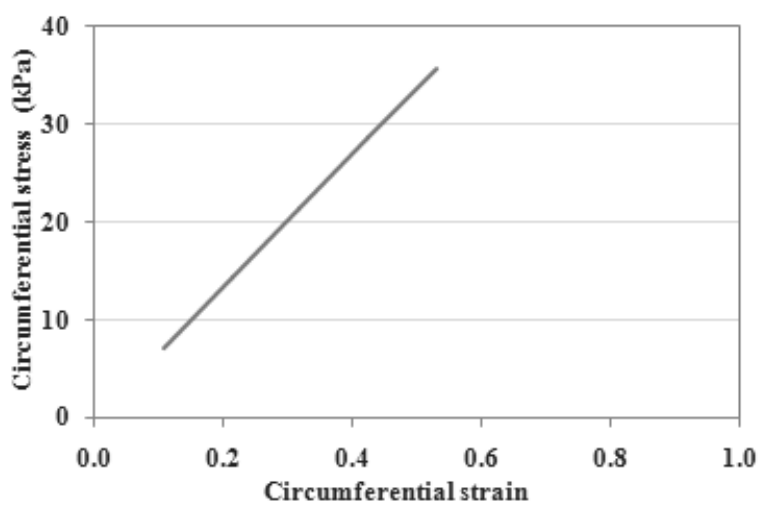

Fig. 10: Circumferential stress-strain relationship

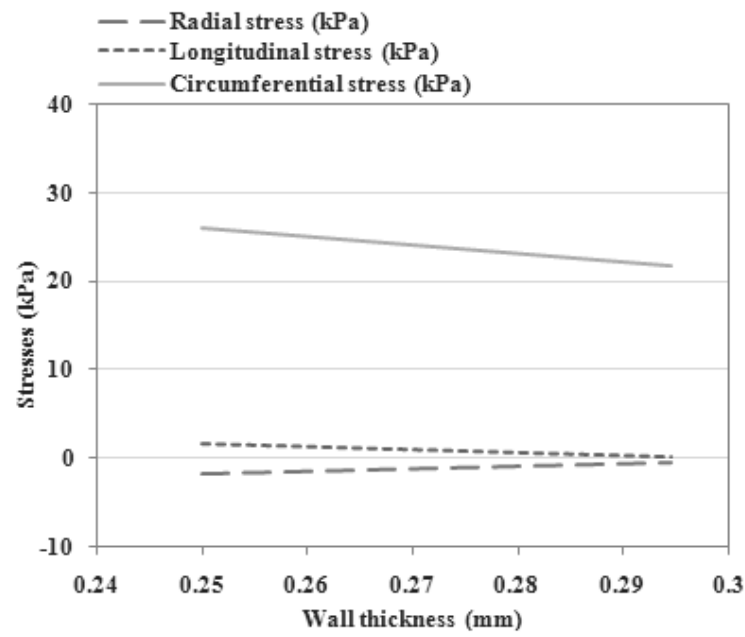

Fig. 11: Radial, longitudinal and circumferential stress distributions across wall thickness at mean pressure of $96.4 \mathrm{mmHg}$ of mice 
Effect of internal pressure on inside diameter, outside diameter and wall thickness, effect of internal pressure on circumferential strain, effect of internal pressure on lumen cross-sectional area and effect of internal pressure on radial stress, longitudinal stress and circumferential stress were shown in Fig. 6-9, respectively.

Also, it can be seen circumferential stress-strain relationship in Fig. 10. Moreover, radial, longitudinal and circumferential stress distributions across wall thickness at mean pressure of $96.4 \mathrm{mmHg}$ of mice was shown in Fig. 11.

\section{DISCUSSION}

From the results, it was found that effect of internal pressure on inside diameter, outside diameter as Fig. 6 had the same trend to results of Guo and Kasseb (2003). Although present results had a little difference with results of Guo and Kasseb (2003) but these diameters had been the same order of magnitude. These show that this simulation was shown good agreement. From this result, it can be seen that, when internal pressure increased, both of inside diameter and outside diameter linearly increased. But, increasing of inside diameter with internal pressure was greater than increasing of outside diameter. This difference of increasing of inside diameter and outside diameter resulted in wall thickness (wall thickness was computed as the difference between the outside and inside radius of vessel at different pressure) reduction linearly with internal pressure as shown in Fig. 6. Again, in this trend of wall thickness reduction of present result, it was also seen in result of Guo and Kasseb (2003) too.

Moreover, the variation of the average circumferential strains with pressure was computed. As internal pressure resulting in increasing of both diameters, it also resulted in linearly increasing of circumferential strain of abdominal wall as shown in Fig. 7.

The variation of lumen cross-sectional area with pressure of abdominal aorta was found to be linear as shown in Fig. 8. Present result of effect of internal pressure on lumen cross-sectional area had the same trend to result of Guo and Kasseb (2003) but there had more difference because lumen cross-sectional area of abdominal aorta increased by square of internal diameter at that internal pressure.

Hence, it can be stated that the linearity between pressure and various metric parameters (diameter, wall thickness, lumen cross-sectional area) in a large range of pressure $(30-150 \mathrm{mmHg}$ ) was remarkable. The wall thickness decreased, whereas the diameter, lumen cross-sectional area increased.
The compliance can be expressed in terms of crosssection area. The result of the mouse cross-sectional area compliance was shown as slope of lumen crosssectional area and internal pressure in Fig. 8.

The variations of radial stress, longitudinal stress and circumferential stress of abdominal aorta with internal pressure are shown in Fig. 9. Similarly, the stresses varied linearly with pressure in the pressure range of 30-150 mmHg. In Fig. 9, it can be seen that the magnitude of radial stress, longitudinal stress and circumferential stress increased with increasing internal pressure. And, from Fig. 11, these values were largest at the inside surface. The radial stress was of course compressive and varies from a value equal to the negative of the internal pressure at the inside to zero at the outside.

Relationship of circumferential stress-strain of abdominal aorta was shown in Fig. 10. This Fig. 10 was shown that stress in circumferential direction linearly increased with strain in circumferential. Slope of this stress-strain was elasticity of abdominal aorta of $70 \mathrm{kPa}$ which was a constant as assumed previously.

\section{CONCLUSION}

Stress-strain analysis of abdominal aortic wall in a case of 3D geometry simulation had been simulated using numerical model. This model were verified with experimental data from Guo and Kassab (2003). Good agreement had been obtained.

The wall thickness decreased, whereas the diameter, lumen cross-sectional area increased with internal pressures.

The magnitude of radial stress, longitudinal stress and circumferential stress increased with increasing internal pressure and values were largest at the inside surface. The radial stress was of course compressive and varied from a value equal to the negative of the internal pressure at the inside to zero at the outside.

\section{ACKNOWLEDGMENT}

This research is supported by The Royal Golden Jubilee Ph.D. Program (RGJ) under The Thailand Research Fund (TRF).

\section{REFERENCES}

Bergel, D.H., 1961. The static elastic properties of the arterial wall. J. Physiol., 156: 445-457. http://jp.physoc.org/content/156/3/458.full.pdf+ht $\mathrm{ml}$ 
Boresi, P. and K.P. Chong, 1961. Elasticity in Engineering Mechanics. 2nd Edn., A WilyInterscience publication, USA.

Carroll, W.F., 1998. A Primer for Finite Elements in Elastic Structures. Wiley and Sons, USA., pp: 512.

Darly L.L., 2007. A First Course in the Finite Element Method. 4th Edn., Thomson Canada.

Guo, X. and G.S. Kassab, 2003. Variation of mechanical properties along the length of the aorta in C57bl/6 mice. Am. J. Physiol. Heart Circ. Physiol., 285: 2614-2622. http://ajpheart.physiology.org/cgi/reprint/285/6/H2 614.pdf

Humphrey, J.D. and S. DeLange, 2004. An Introduction to Biomechanics Solid and Fluid. Analysis and Design. Springer, pp: 631.
Silver, F.H., P.B. Snowhill and D.J. Foran, 2003. Mechanical behavior of vessel wall: A comparative study of aorta, vena cava and carotid artery. Ann. Biomed. Eng., 31:793-803. DOI: 10.1114/1.1581287

Zhao, J., J. Day, Z.F. Yuan and H. Gregersen, 2002. Regional arterial stress-strain distributions referenced to the zero-stress state in the rat. Am. J. Physiol. Heart Circ. Physiol., 282: 622-629. PMID: 11788411 